\title{
Enhancing the End-to-end Link Performance of Traditional Satellite Ground Networks via Software- Defined Networking Controllers (POX/RYU)
}

\author{
Ehab M. El Sabbagh \\ Military Technical College \\ Cairo, Egypt
}

\author{
Mohamed Hussein \\ Military Technical College \\ Cairo, Egypt
}

\author{
Ashraf D. Elbayoumy \\ Military Technical College \\ Cairo, Egypt
}

\begin{abstract}
Nowadays, the satellite networks with communication systems of terrestrial network undergo an augmentation of traffic flow which is one of our main issues. We oppose in vertical integration of the traditional networks a usual inflexible and inefficient quality of services' (QoS) parameters as, latency and throughput. Recent technologies are rapidly being emerged and deployed, those are software defined networking (SDN) with its complementary, network function virtualization (NFV), additionally network virtualization (NV) which masks network resources with more flexibility in managing network via advanced programmability and OpenFlow controllability. Our proposal in this state of paper is a new programmable and centralized satellite network architecture based on SDN/NFV, composing of SDN layers that will correspond the functionalities of traditional devices in a virtualized behavior with a comparative performance results between OpenFlow controllers (POX/RYU) and traditional network to introduce enhanced services over satellite ground networks integrated through terrestrial networks. We disseminate our contribution regarding end-to-end (E2E) link performance and QoS parameters as latency calculation and throughput bandwidth-on demand by implementing different tests using "Mininet" open source network simulator and emulator tool to enhance augmentation of legacy in network's link evaluations via OpenFlow controllers' (RYU/POX).
\end{abstract}

\section{General Terms}

Controller, SDN, Satellite Networks, Algorithm.

\section{Keywords}

Software-defined networking; Network virtualization; OpenFlow; Software defined satellite network; POX/RYU controllers

\section{INTRODUCTION}

The satellite system is constructed upon the integration between space-segment networks and ground-segment networks exited to terrestrial backbone network. The conventional satellite and classical terrestrial networks are still suffering from inflexibility and adaptability in servicing satellite networks for different vendors preserving their own specific interfaces, this leads to inefficient delivery of services that are necessary to execute network functions on satellite station networks. The decentralized management of the network embodied by the inefficient management of non-utilized resources and static routing algorithms, alongside the complexity of network configuration lead to inefficient traffic schedule with severe dynamic configuration overall the ground satellite stations networks. As a result, software defined networking (SDN) [1-3] and network function virtualization (NFV) [4-6] embracing the vision and concept of centralization with logical control to face hard challenge in improving quality of services' (QoS) parameters and performance of such networks. Furthermore, programmability and virtualization of network are very incentive for updating network architecture and developing the traditional features of satellite network by separating the control plane of all devices interconnected to the network from data plane to overcome the limitations exposed by current legacy networks.

In this paper, we focused on the additional advantages that have been introduced and applied by SDN controllers (POX/RYU) on end-to-end (E2E) links of satellite ground segment/station networks, which are operated by satellite network operational center (SNOC) controlled by satellite operator (SO) that has a main role in managing the whole satellite and selling bandwidth capacity at satellite transponder level to several satellite network operator (SNO). SNOC has resilience functionality in connecting between multiple of satellite network control centers (SNCCs) for different ground stations and utilizing the centralization of satellite management via satellite network management center (SNMC) which is responsible in routing policies calculation, security and management strategy of resources.

Moreover, software defined satellite network (SDSN) is deployed for logical flow entries of the network to improve the forwarding decisions supported by the controller which runs the software developed programmable code scheme. Herein, our proposal we will suggest a specific virtual and programmable correspondence of satellite's network devices (SNCC, SNMC) in the designed framework of SDN layers architecture to earn all the advantages of virtualization by SDN/NFV on satellite hubstations and ground segment. In this paper we will improve the performance results of E2E link between different nodes of a traditional network topology through OpenFlow network based on (POX/RYU) controllers with a comparative performance enhancement applied upon "POX" to a previous related work. Software Defined Satellite Network (SDSN) was introduced in [7], showing the challenges and benefits of applying SDN on traditional and satellite networks. Most of the previous work focused on the theoretical model of SDN/NFV-based satellite networks. The contribution in this paper is to improve link performance and QoS metrics such as latency and throughput data rate of a certain link between different nodes with an enhancement in bandwidth related to the network using a modified POX controller codes, also we will separate the control planes of all infrastructure devices in satellite network (SNMC, SNCC,....) to centralize it in a unified controller to carry out the same roles of functions but in a virtualized way correspondent to the traditional devices of satellite legacy network as in Fig.1.

The rest of the paper is organized as follows; Section 2 reviews the background of SDN/NFV as a concept technique and related work. Section 3 describes our proposal for corresponding SDSN 
Structure prototype framework to traditional satellite network topology [8]. Section 4 Section shows the results of the simulation test on links' performance evaluations between different nodes. Section 5 conclusion of what proposed in this paper. Section 6 for our future work.

\section{BACKGROUND AND RELATED WORK}

\subsection{Background}

By adopting SDN and NFV techniques of network technologies in satellite communications networks, satellite networks and terrestrial networks begin to face challenges of enhancing scalability, network performance, traffic schedule, flexibility of controlling and management, dynamic of routing configuration and calculation, and utilizing resources management. SDN and NFV can evolve the satellite architecture to fulfill all previous issues with high-adaptability and interoperability of heterogonous networks. As, it provides seamless integration between satellite hub-stations and terrestrial networks with sufficient improvement in applications of satellite network. Satellite networks embracing SDN with its three main layers (management plane layer, control plane layer and data plane layer ), virtual functionality allows nodes to establish advanced programmable operational layers and introduce some abstraction layers as hypervisor accessed via application programmable Interfaces (APIs) as shown in Fig.1.

The network policies are defined in the management plane and introduced them to the lower layer (control layer) by means of APIs to establish forwarding decisions that settled in data plane which forwarding data accordingly. NFV is a complement to SDN logical network to assign functions upon virtual nodes with virtual links.SDN open a new framework of standards and protocols as "OpenFlow protocol" [9]. SDN separates between the control plane (forwarding decisions plane) and data plane (execute decisions plane) of the network's nodes connected and raised up a new entity called "controller" that implements a centralized control and management for multiple network nodes, as depicted in Fig. 2.With the centralization management and controlling, the global overview of satellite ground segment/station network will implement the simplest forwarding decision and optimize routing strategies with flexibility in configuration functions of hardware $(H / W)$. It facilitates programmability and setting up of virtual machines (VMs) that process and provide physical servers (nodes), improving the ability of processing in VMs (i.e. virtual switch, virtual router, virtual gateway) that are allocated for hubstations of ground segment by multiple resources. Multiple virtual nodes are abstracted network devices carried on single physical node to perform NFV. In other words there is an abstraction sub-layer called "Hypervisor" which is the virtual machine monitor between $\mathrm{H} / \mathrm{W}$ and $\mathrm{VMs}$ illustrated in Fig.1.NFV decouples network functions such as routing, firewalling, intrusion detection, and NAT (network address translation) from $\mathrm{H} / \mathrm{W}$ platforms and implements these functions in software. It utilizes standard virtualization technologies that run on high-performance hardware to virtualize the network functions.

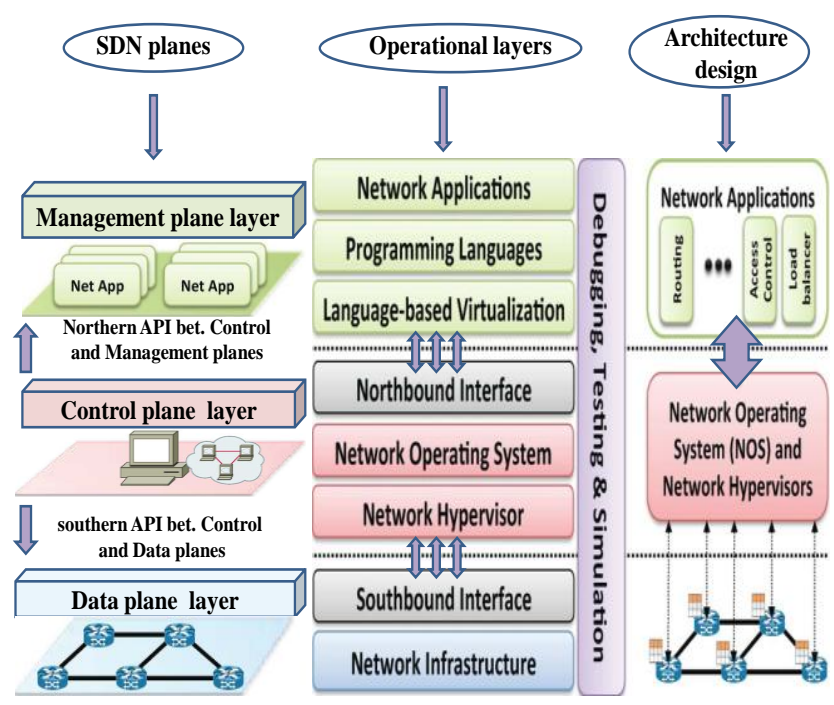

Fig. 1. SDN architecture layers (Planes, Operational layers, Design architecture)

\subsection{Related work}

In recent researches authors try to get new methods to adopt SDN concept and NFV technologies to design a new state-of-art in satellite networks. Hereby, some proposed articles as Smart gateways were discussed in [10], illustrated the usage of site diversity technique and forwarding to exploit and realize the antenna pooling scenario between different sites to improve high bandwidth capacity of multimedia satellite communications. In [11], satellite networks were provided with high efficiency, finegrained control, as well as flexibility using OpenSAN. In [12], broadband satellite networks were discussed, clarifying how SDN/NFV with network virtualization enhanced the satellite network services in exchanging information between sites using diversity techniques.

Our objective in this paper to get a new way to increment the efficiency of QoS parameters with respect to delay and throughput to attain high performance via satellite ground segment network by inspiring a comparative relation between frameworks, the main issue in [13], that authors presented a simulation using simple six nodes connected by a certain topology to enhanced time delay between nodes and throughput capacity accordingly, compared to a traditional strategy. Actually we single out a convenient emulator to obtain a precise enhanced evaluation results for all network protocols used around, tends to improve the previous related work results in both delay and throughput of network payload utilizing the default open source simulator for the recent versions of "Mininet" and "Miniedit" tools version 2.2.2 with OpenFlow versions v.1.5, v.1.6 to imitate the real time of the network.

In [14], mainly spots on the using of SDN technology for the viability of internet in advance for the future, presented that by 'Mininet' to implement an OpenFlow interface to his network simulation framework connected by POX controller to verify the efficiency of bandwidth between network's nodes and realize the adequacy of using switch component linked by POX, but we will mainly focus on modifying the features of POX controller to attain better performance and increase the efficiency of the controller that will aid us to have higher performance particularly for bandwidth between nodes to meet the overrun of network requirements. 


\section{DEFINED SATELLITE NETWORK STRUCTURE PROTOTYPE TOPOLOGY}

We will focus in this part on how SDN/NFV is appended to SDSN [15]. SDSN distributes the routing tables and the configurations' instructions through inter satellite links (ISLs) and interconnected links (ILs) with high performance of throughput and low latency between links by means of POX/RYU controllers. SDSN is controlled by a layer based-on OpenFlow protocol, forwarding flow tables between infrastructure nodes.

\subsection{Management /Application plane}

We deploy a virtualized SNMC instead of another in a traditional satellite hub station network to be responsible for managing and controlling network resources embedded in traditional satellite SNMC. It will avail in improving the management services [16] [17] by utilizing the centralization of SDN controller to translate the upper layer instructions from SNOC.

This centralization is taking charge of routing algorithms between nodes and dedicates routing paths according to the status of the satellite network, gathered by space segment transponder, and controlled by SNOC to exchange information between satellite terminals (STs).Flowvisor interface is applied between POX and RYU controllers, it acts as virtual proxy that control OpenFlow packets between switches and controllers for the whole network. It partitions the link bandwidth and flow tables entries of each switch of the entire network designed as in Fig.2. It is deployed here between POX and RYU controllers [18] to admit multiple controllers to be familiar with each otherand allow them to share the same OpenFlow networking devices.

SNOC provides management of resources through SNMC, so virtualization adds a higher level of services of satellite access via SNOC with more scalable and flexible management resources. Moreover, SNMC performs hub-stations' configurations and security management of the nodes as, gateways (GWs), performance enhancement proxy (PEP), point of presence (PoP) and, STs. PEP enhanced the TCP performance over SLs, while $\mathrm{PoP}$ is an interface between different communicated network entities as in Fig.3. So, virtualization of SNMC will introduce the flexibility in management of functions and resources. We deploy controller centers SNOCs (POX controller) linked by SNCCs that are connected with their hub station $1 \mathrm{~A}$ and hub station 1B respectively. SNOC deployed in satellite network to have all the status information from the ground stations, it has an overview on the whole satellite network segments (ground and space) accordingly.

The hub stations will facilitate controlling on satellite forward link transmitted unit (FL-TU) and return Link Reception Unit (RL-RU) by means of GWs. SNOC acts as an interface to the satellite operator (SO) in satellite network. The requirement of satellite virtual network operator center (SVNOC) services is obvious, as VNOC services will allow flexible management over satellite resources that will be shared between multiple of SVNOCs by dedicating the bandwidth needed to guarantee QoS parameters. SNOC presents all management services on virtualized hub station's components by their SVNOCs embedded in by adding management policies required showed in Fig. 2.

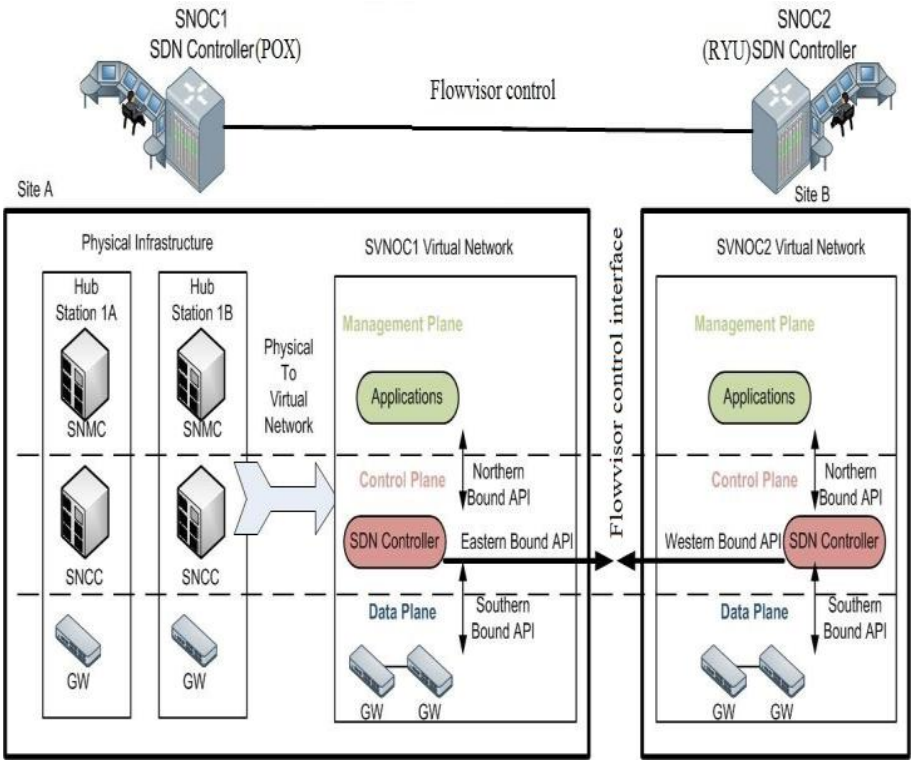

Fig. 2. Description of physical to virtual functionality of SNOC in SDN.

\subsection{Control plane}

SNCCs are assigned to provide control functions and carry out the STs' control admissions and specifications of forward and return links. For more practical clarity, we follow up the real structure of satellite ground stations including its hub-stations interconnected externally by the terrestrial network, even through point of presence (PoP) located at internet exchange points illustrated in Fig. 3. We designed a simple proposal between the two sites (A, B), the SDSN structure prototype that each composed of:

3.2.1 Space segment transponder (GEO or MEO or LEO) linked with end user node (ST) via user link UL and linked to SOC node via control link (CL).

3.2.2 The SOC connected by IL1 \& IL2 with two different SNOCs nodes respectively, that SDN controllers, POX controller, RYU controller are embedded inside for resilience and security issues.

3.2.3 The network status collection and SDN managing controller are designed in both POX and RYU controllers. The two SDN controllers are connected to each other and distributed by means of Flow-Visor controller interface (East/West API's) allows multiple logical networks share the same OpenFlow networking infrastructure.

The functions of these interfaces include import and export data and information between POX and RYU controllers to fulfill interoperability of the entire network. Hereby, we enforce the variety of platforms, to increase rigidity by reducing the appearance of common faults of S/W between controllers.

SNOC node (POX/RYU) operates to centralize management services and status information by means of virtual SNMC and centralize controlling network overview by virtual SNCC in the same hub station (hub station $1 \mathrm{~A} / 1 \mathrm{~B}$ or $2 \mathrm{~A}, 2 \mathrm{~B}$ ), so we put them under the control of SNOC to have global overview network for flexibility. 


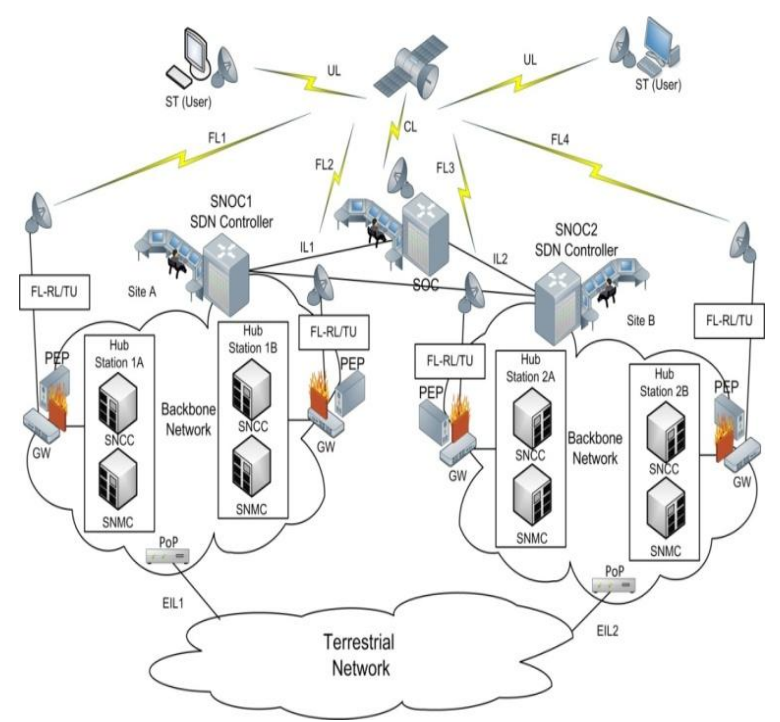

Fig.3. SDSN prototype topology

\subsection{Data/Infrastructure plane}

Moreover, we deployed the GWs to act as OpenFlow switches via the backbone network to provide the diversity case between stations, whether internally between hub stations (antenna pooling case) or externally between sites and terrestrial network via PoP. GWs adapts up and down satellite linking through FLTU/RL-TU to communicate with space segment via feeder links (FLs). Our objective is resilience in service delivery within this plane, so we are embedding several service functions as, firewall, NAT and virtual private network (VPN) proxy in VMs to be deployed over network's GWs.

\section{SIMULATION OF THE NETWORK WITH EVALUATION RESULTS 4.1 The improvement of bandwidth capacity between nodes}

Here we constructed the test bed for our experimental results by deploying virtual machine to validate the soundness of the related work mentioned above [14], We proposed a scheme as illustrated in fig. 4 which has been applied specifically upon POX connected by three hosts $(\mathrm{H} 1, \mathrm{H} 2, \mathrm{H} 3)$; by creating entry table for all connections of SDN topology. This table is utilized to preserve all IP addresses of the entire packets transmitted .Host IP address (IPh) specified for each host which has a counter $\mathrm{Cn}$ loading the packets per each flow. The created entry table has its own counter $\mathrm{Ct}$ to state the frame of packets transmitted per each flow .Cd for counting the duration of time which the flow entry should spend in the flow table otherwise it will drop the entry information and request for a retransmission to grantee that no congestion will be occurred and avoid the buffer queuing to take place in this process, finally we improved the utilization of BW between all nodes linked by valuable performance for POX controller as in fig .5 and provided BW capacity of POX controller between all hosts included in our network to ensure the efficiency of the connected OpenFlow switch and facilitate observation between controller and the switch.

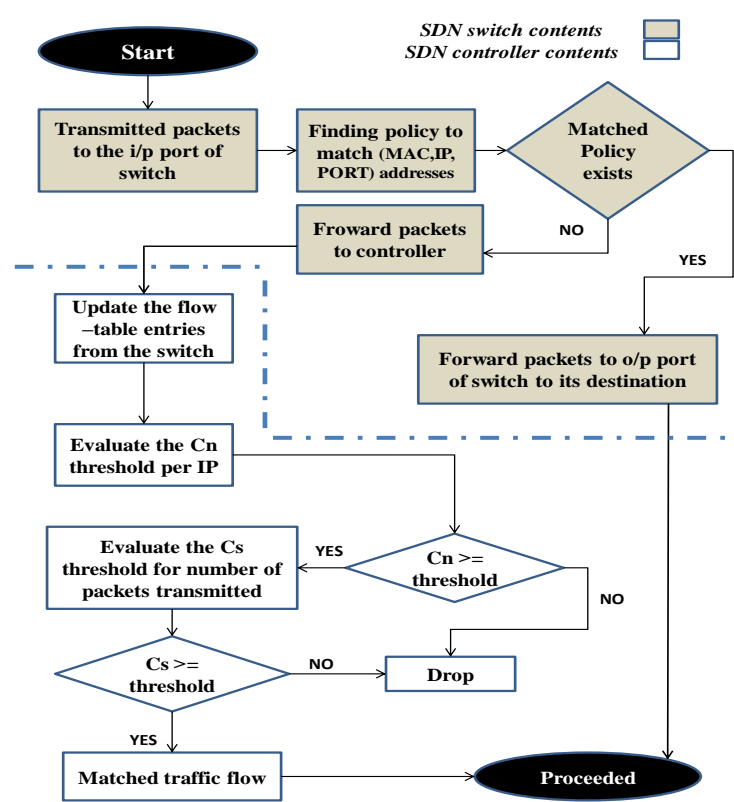

Fig.4 The proposed flowchart for POX controller packets flow

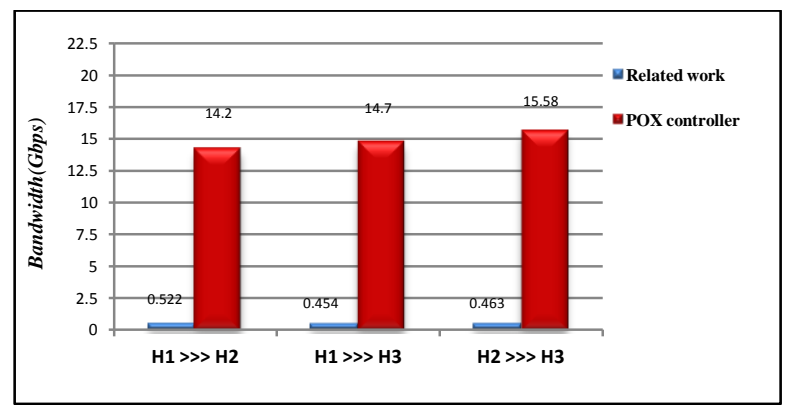

Fig. 5. Comparative BW between different nodes with related work using POX controller

\subsection{The improvement of performance of controllers regarding delay and throughput}

The established system consists of two main components:- First, virtualized devices to the whole ground segment infrastructure network which include the OpenFlow switches and end-users, compiled over "Miniedit" tool, second, connected with POX/RYU controllers, to overview the entire network design. The most favorable E2E parameters of network transmission performance are throughput and delay [19]. It is desired to be satisfactory with the existed current applications and future usage of satellite internet protocol (IP) networks. The ground satellite links are adhered by delay and throughput data rate, the performance of applications using TCP/IP is so sensitive accordingly. So, a comparative analysis for performance and QoS parameters (BW, delay, reliability ...) has been proposed between accustomed network topology and OpenFlow controllers (RYU/POX) topology by performing a pining test between different nodes. Also we attempt to improve results of QoS metrics over related previous work mentioned in [13] by a comparative usage of 6 linked different nodes for ground segment of satellite utilizing the simulation tool "Mininet" to emulate the network transmission delay and throughput execution analyzed. We acquired that by mainly two situations: 


\subsubsection{Link delay between two nodes}

The Round Trip Time (RTT) calculated for satellite links is based on latency impact, so, to attain accurate performance with high QoS we should reduce delay as much as possible between nodes.RTT express $100 \%$ of latency contribution on E2E satellite link between nodes. Herein, we specified physical links connected between nodes with default BW of "Mininet" simulation tool [20-21] in (Gbps) and with latency delay (1 ms) for all network links to obtain 100 trial for 50 packets per flow to validate the soundness of the related work [13] that are transmitted from one node to another by performing internet control message protocol (ICMP) querying messages for echo and reply requests between nodes linked to each other, Also our contribution in enhancing the evaluations of previous related work mentioned above by observing the transmission of 150 packets over the links between different nodes of our proposed network as in Fig.6.

\subsubsection{Link throughput between two nodes}

we investigated the data rate capacity (throughput) transmitted via a candidate link [22] by comparing the same previous accustomed related network topology and OpenFlow SDN

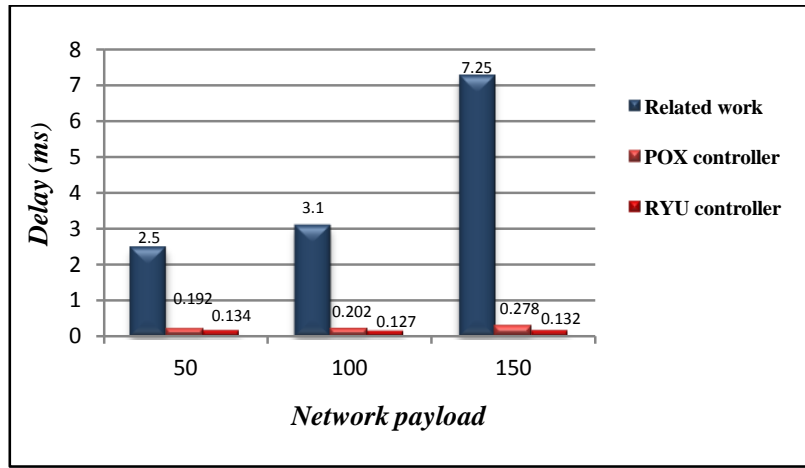

Fig.6 Comparative results of network delay between POX/RYU and related work

controllers (RYU/POX) topology network links utilized in analyzing throughput parameter as depicted between nodes' link response in Fig. 7 and give the performance comparative results between the usage of SDN controller (POX/RYU) and the previously mentioned related work is shown in Fig .7 and to define the amount of transmitted data rate in a given time.

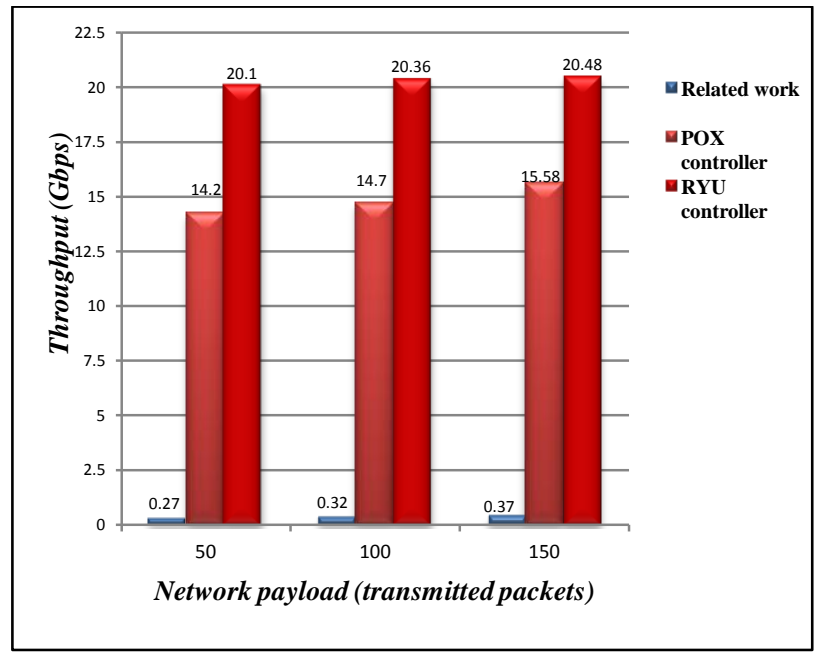

Fig.7 Comparative results of network throughput between POX/RYU and related work

\section{CONCLUSION}

In this paper, we spotted on the network concept of SDN/NFV with network virtualization of network technologies for satellite networks and the privileges gained on traditional satellite networks to face most of the recent challenges and enhance its scalability, as well as the function of SDN controller and OpenFlow stat of art. We detailed the structure of SDSN and the interfaces between the planes of SDN architecture and the correspondence for physical nodes as, SNCC and SNMC which carry out the same functionality by programmability and virtualization mapping. We proposed a virtualized correspondent architecture design based on SDN/NFV to a traditional network to introduce enhanced services over satellite ground networks integrated with terrestrial networks. The SDN/NFV supports all developments of switching and routing capabilities to enhance services and status of network. In this paper we assumed an algorithm over POX controller to improve its performance in comparison with a related work enhancing $\mathrm{BW}$ between nodes accordingly.

Also, we investigated a comparison between E2E link performance analysis for throughput and delay in comparison to related work regarding the switch component and OpenFlow controllers (POX/RYU) networks environment with improvement of those QoS parameters against previous related work. We contributed our results for RTT and throughput OpenFlow entries to be more efficient when SDN controllers (POX/RYU) is used in real time circumstances.

\section{FUTURE WORK}

We will attempt to extend a new way to optimize higher performance and efficiency for RYU and POX controllers by adding policies and management decisions over SDN structure and applying machine learning algorithms on controllers with respect to TCP and UDP flows, by implementing ns2 and ns3 network emulators for SDN networks, to acquire the best efficient BW between different nodes connected to OpenFlow switch accompanied by better performance of QOS parameters.

\section{REFERENCES}

[1] Nunes, B., Mendonca, M., Nguyen X., and Obraczka, K., "A survey of software-defined networking: Past, present, and future of programmable networks," ", Communications Surveys \& Tutorials, IEEE, vol. 16, pp. 1617-1634, Feb. 2014.

[2] Kreutz D., Ramos FM., Verissimo PE., Rothenberg CE., Azodolmolky S., Uhlig S."Software-defined networking: A comprehensive survey," Proceedings of the IEEE, vol.103, pp.14-76, Jan. 2015.

[3] S. Azodolmolky, "Software defined networking with OpenFlow,", $1^{\text {st }}$ ed,2013, pp. 15-21.

[4] Nadeau, L., Thomas, D., and Ken, G., "SDN: Software Defined Networks: An Authoritative Review of Network Programmability Technologies", $1^{\text {st }}$ ed., O'Reilly, Aug 2013, pp.207-233.

[5] Mijumbi, R., Serrat, J., Gorricho, J., et al., "Network Function Virtualization: State-of-the-art and Research Challenges", Communications Surveys \& Tutorials, IEEE, Sep. 2015 vol. 18, pp. 236-262.

[6] Stallings, W., "Foundations of modern networking: SDN, NFV, QoE, IoT, and Cloud", 2nd ed., Addison-Wesley Professional, 2016, pp.177-223.

[7] Tang, Z., Zhao, B., Yu, W., Feng, Z., Wu, C. "Software defined satellite networks: Benefits and challenges", In 
Computing, Communications and IT Applications Conference (ComComAp) IEEE, pp.127-132, Oct. 2014.

[8] Li, T., Zhou, H., Luo, H., Xu, Q., Ye, Y. "Using SDN and NFV to implement satellite communication networks," In Networking and Network Applications (NaNA), 2016 International Conference, pp.131-134, Jul. 2016.

[9] McKeown, N., Anderson, T.E., Balakrishnan, H., Parulkar, G.M., Peterson, L.L., Rexford, J., Shenker, S., \& Turner, J.S. "OpenFlow: enabling innovation in campus networks," ACM SIGCOMM Computer Communication Review, vol.38, pp.69-74, Mar. 2008.

[10] Rossi, T., Maggio, F., De Sanctis, M., Ruggieri, M., Falzini, S., Tosti, M. "System analysis of smart gateways techniques applied to Q/V-band high throughput satellites," In Aerospace Conference, IEEE, pp.1-10, Mar. 2014.

[11] Bao, J., Zhao, B., Yu, W, Feng, Z., Wu, C., Gong, Z. "OpenSAN: a software-defined satellite network architecture," In ACM SIGCOMM Computer Communication Review, vol. 44, no.4, pp. 347-348, Aug. 2014.

[12] Bertaux, L., Medjiah, S., Berthou, P., et al., "Software defined networking and virtualization for broadband satellite networks", IN Communications Magazine IEEE, vol. 53, pp. 54-60, Mar. 2015.

[13] Wang, C., \& Yu, X. "Application of virtualization and software defined network in satellite network," In 2016 International Conference on Cyber-Enabled Distributed Computing and Knowledge Discovery (CyberC), IEEE, pp. 489-493, Oct. 2016

[14] Prete, Ligia Rodrigues, Ailton Akira Shinoda, Christiane Marie Schweitzer, and Rogério Leão Santos de Oliveira. "Simulation in an SDN network scenario using the POX Controller." In 2014 IEEE Colombian Conference on Communications and Computing (COLCOM), pp. 1-6, Jun. 2014.

[15] Ferrús, R., Koumaras, H., Sallent, O., Agapiou, G.,
Rasheed, T., "SDN/NFV-enabled satellite communications networks: Opportunities, scenarios and challenges", In Physical Communication, vol. 18, pp.95-112, Mar. 2016.

[16] Hyojoon, K. and Feamster, N., "Improving network management with software defined networking", In IEEE communications Magazine, vol. 51, no. 2, pp. 114-119, Feb. 2013.

[17] Li, T., Zhou, H., Luo, H., You, I, Xu, Q. "SAT-FLOW: Multi-strategy flow table management for software defined satellite networks", IEEE Access, Vol.5, pp. 14952-14965, Jan 2017.

[18] Shalimov A., Zuikov D., Zimarina D., Pashkov V., Smeliansky R. "Advanced study of SDN/OpenFlow controllers" In Proceedings of the 9th central \& eastern european software engineering conference in Russia, Oct. $2013,10.1145 / 2556610.2556621$

[19] Ferrús, R., Sallent, J., Ahmed, T., Fedrizzi, R. "Towards SDN/NFV-enabled satellite ground segment systems: Endto-End Traffic Engineering Use Case", IEEE International Conference on Communications Workshops, ICC Workshops, Paris, France, May 2017, 10.1109/ICCW.2017.7962771.

[20] Lantz. B and O'Connor. B. "A Mininet-based virtual test bed for distributed SDN development", ACM SIGCOMM Computer Communication Review, vol.45, no. 4, pp.365366, Sep. 2015.

[21] Bholebawa IZ, Jha RK., Dalal UD. "Performance analysis of proposed OpenFlow-based network architecture using Mininet". In Wireless Personal Communications, vol. 86, no.2, pp. 943-58, Jan. 2016.

[22] Rossi T, De Sanctis M, Cianca E, Fragale C, Ruggieri M, Fenech H., "Future space-based communications infrastructures based on high throughput satellites and software defined networking", In Systems Engineering (ISSE), IEEE International Symposium,pp.332337,Sep.2015, 10.1109/SysEng.2015.7302778 